\title{
REPRESENTACIONES DE LA ININTERRUMPIDA Y GROTESCA CELEBRACIÓN CARNAVALESCA EN DOS UTOPÍAS DE LA ALEMANIA DEL SIGLO XVII
}

\author{
Júlia Ciasca Brandão \\ (Universidade Estadual de Campinas, Brasil)
}

\section{RESUMEN}

En el año 1640 se publicó Utopía, obra homónima a la de Tomás Moro, escrita por Jakob Bidermann, jesuita alemán e inquisidor de la Iglesia Católica en Roma. El autor, miembro activo de la cultura tridentina, escribió el texto en latín con la finalidad didáctica de enseñar ejemplo y moral para los alumnos del colegio de los jesuitas en la ciudad de Augsburgo. En 1677, Christoph von Andreas Hörl Waltersdorf, de quien poco se sabe, tradujo el texto del latín al alemán y lo publicó bajo el título de Bacchusia oder Fastnachtland. A excepción de algunas diferencias entre la obra original y su traducción, el texto se refiere al viaje de tres amigos a un país imaginario donde los habitantes celebran un carnaval eterno, el caos es institucionalizado y se suspenden las reglas de buena conducta.

PALABRAS CLAVE: Utopía, Goliardía, Carnaval, Guerra de los Treinta Años.

\section{REPRESENTATIONS OF THE UNINTERRUPTED AND GROTESQUE CARNIVAL CELEBRATION IN TWO UTOPIAS OF SEVENTEENTH-CENTURY GERMANY}

\begin{abstract}
In 1640, Utopia, the homonymous work of Thomas More, written by Jakob Bidermann, German Jesuit and Inquisitor of the Catholic Church in Rome, was published. The author, an active member of the Tridentine Culture, wrote the text in Latin with the didactic purpose of teaching moral example to his students at the Jesuit College in the city of Augsburg. In 1677, Christoph von Andreas Hörl von Waltersdorf, of whom little is known, translated the text from Latin into German, and published it under the title Bacchusia oder Fastnachtland. Except for some differences between the
\end{abstract}


original work and its translation, the text recounts the journey of three friends to an imaginary land where the inhabitants celebrate an eternal carnival, chaos is institutionalized and the rules of good behavior are suspended.

KEYWORDS: Utopia, Goliardia, Carnival, Thirty Years’ War.

\section{EL PAÍS DEL CARNAVAL}

Cuando Georg Stengel, rector del colegio jesuítico de Dillingen en Baviera, decidió editar y publicar la Utopía de Jakob Bidermann, tenía la intención de que sus lectores vieran una imagen real de sí mismos y del mundo en que vivían ${ }^{1}$. El hecho es que en 1640, gran parte de Europa sufría la continuación de la Guerra de los Treinta Años (1618-1648) ${ }^{2}$. El conflicto político externo e interno, hegemónico, dinástico, territorial y religioso, se acepta hoy como la "catástrofe alemana", porque retrasó aún más la posibilidad de unificación de los trescientos cincuenta estados alemanes y dejó el territorio devastado económica y socialmente. Otto Maria Carpeaux, en la obra $A$ História Concisa da Literatura Alemã (2013), hace una breve evaluación de sus consecuencias:

Todas as potências do continente europeu se bateram em solo alemão sob pretexto de intervir na guerra religiosa entre os católicos e os protestantes da Alemanha. Nunca outro país foi submetido a tão cruel e sistemática devastação, sendo a população, em certas regiões, reduzida à décima parte e sendo destruídos todos os valores materiais e morais. Foi a maior catástrofe da história alemã, da qual sobrou um país paupérrimo, atrasado e politicamente dividido em inúmeros pequenos principados, governados no Norte por mesquinhos régulos luteranos e no Sul por relaxados prelados católicos, enquanto nos poucos Estados maiores se estabeleceu o absolutismo à maneira francesa ${ }^{3}$.

\footnotetext{
${ }^{1}$ En el primer capítulo de la Utopía de Bidermann, uno de los personajes dice una frase que será recordada por Stengel, en la "Epístola Dedicatoria" que precede al texto: «Das Stück war so gut und korrekt, dass die allerbesten Schauspieler es aufführen und alle Welt es ansehen sollte, dann sähe die Menschheit ein naturgetreues Abbild ihrer selbst» [La obra era tan provechosa y correcta, que los mejores actores deberían representarla y todos deberían verla; entonces, la humanidad vería una imagen real de sí misma] (Jakob Bidermann, "Utopia", en Margrit Schuster, Jakob Bidermanns "Utopia": Ed. Mit Übersetzung und Monographie; nebs vergleichenden Studien zum beigedr. Plagiat d. Christoph Andreas Hörl von Wattersdorf („Bacchusia oder Fassnacht-Land“) (Bern: Peter Lang, 1984), 25.

${ }^{2}$ Cf. el estudio de Friedrich Schiller, Historische Schriften und Erzäblungen (Stuttgart: Deutscher Klassiker Verlag, 1879); Jacob Burkhardt, Der Dreißigjährige Krieg (Frankfurt: Surkamp, 1992); entre otros.

${ }^{3}$ Otto Maria Carpeaux, História concisa da literatura alemã (São Paulo: Faro, 2013), 28.
} 
Es este mundo grotesco, que se define por el desorden, el caos y la animalidad -la "imagen real" de los lectores y su mundo-, el que Stengel tenía la intención de publicar. La obra, en verdad, fue escrita en 1604 por Jakob Bidermann, que no conocía el siguiente escenario de guerra. La principal intención del autor, miembro activo de la cultura tridentina, era amonestar e instruir a los estudiantes del colegio jesuítico. Según el rector, los estudiantes vivían en un ambiente goliardesco: atraídos por el libertinaje y por la vida bohemia, practicaban muchas infracciones contra los estatutos de la Compañía, especialmente en los últimos días del Carnaval, cuando bebían demasiado y ejercían violencia de todo tipo ${ }^{4}$. La postura goliardesca (la transgresión de las normas vigentes, los hábitos disolutos y la satisfacción de los placeres inmoderados) era común entre los estudiantes de las universidades europeas desde el siglo XII, y marcaba su transición de la vida universitaria irresponsable a la edad adulta. Esto se intensificaba en el período de Carnaval.

Bidermann, siguiendo la tradición de los relatos de exempla, deseaba iluminar los vicios y demostrar las consecuencias de una rutina de excesos en un mundo imaginado. Aunque alimentado por una intención didáctica y seria, su texto -lleno de farsas, anécdotas y aventuras- no pierde la tendencia irónica y satírica, que se caracteriza por el ridendo dicere verum, extendido desde la Edad Media ${ }^{5}$. Desde 1604, su obra era leída en las clases y copiada por los estudiantes que, según Stengel, eran «capturados por la «magia de los cuentos» ${ }^{6}$.

No obstante la fascinación que había producido, el manuscrito de Bidermann fue editado y publicado oficialmente sólo en 1640, por Stengel. En 1677, la obra de Bidermann fue traducida del latín al alemán por Hörl. Sin embargo, su traducción tiene diferencias sustanciales con respecto al texto original. Quienes se dedicaron al estudio de la Utopía de Bidermann y se toparon con esta versión alemana, tuvieron dificultades para clasificarla como traducción libre, paráfrasis o imitación del texto del jesuita. Bacusia traduce la estructura de la narrativa de Utopia -el viaje de tres amigos alemanes al país del Carnaval-; pero Hörl no tradujo algunos pasajes presentes en el texto original y llevó diversos elementos de la Utopía a su propio entorno histórico y temporal. Por otra parte, los objetivos y el público de los autores son otros. Mientras Bidermann fue movido por una intención amonestadora y escribió el texto para sus alumnos, Hörl, en la introducción de su obra, explicó que Bacusia fue escrita para entretener al lector y pasar el tiempo. A pesar de las diferencias, la Utopía de Bidermann y su versión alemana son la representación de un mundo carnavalesco y grotesco, que

${ }^{4}$ La postura goliardesca (la transgresión de las normas actuales, los hábitos disolutos y la satisfacción de los placeres inmoderados) era común entre los estudiantes de las universidades europeas de los siglos XII y XIII y marcaba su transición de la vida universitaria irresponsable a la edad adulta. Esto se intensificaba en el período de Carnaval. Sobre los goliardos y la literatura goliardesca, cf. Jacques Le Goff, Os Intelectuais na Idade Média (São Paulo: Editora Brasiliense, 1989).

${ }^{5}$ «Der feine, aber scharfe Witz [ist] bereits einen Hinweis auf die spöttische-satirische Tendenz des Romans. Auch der im Untertitel aufgeführte Doppelbegriff ludicra et seria, welcher seit dem Mittelalter den Stil des ridendo dicere verum kennzeichnete, verdeutlicht dasselbe». Schuster, 1984, 25.

${ }^{6}$ «Durch das überaus geschliffene Latein und den Zauber der Geschichten vermag er [Bidermann] den Leser (...) zu feßeln (...)». Stengel, Apud. Schuster, 1984, A3. 
se define por el desorden, el caos y la animalidad, que Stengel en 1640, quiso publicar en medio de la oscuridad de la Guerra de los Treinta años.

\section{LA GUERRA DE LOS TREINTA AÑOS}

Esta guerra fue considerada por muchos la «guerra de las guerras», debido su larga duración. En efecto, la experiencia de una guerra aparentemente interminable, llena de sufrimiento, hambre, enfermedad y destrucción, en general, permitió la composición de varios textos literarios en los que se unieron la conciencia de la muerte, la inestabilidad de la fortuna y la debilidad del tiempo, esto es, percepciones barrocas de la vida. En un momento como este, se vivía constantemente bajo el signo de la muerte. Hasta hacía pocas décadas, el mundo todavía parecía perfectamente inteligible e dominable, porque las jóvenes ciencias naturales habían empezado a desentrañar sus misterios a través de la obra de Copérnico, Galileo, Kepler y Paracelso. Y, de repente, este mundo se reveló una máquina feroz cuyos engranajes empezaran a aplastar y destruir implacablemente a culpables e inocentes. Esta es la causa del terror, del miedo existencial, de la sensación de estar completamente dejado al azar, de ser un juguete de la fortuna inconstante, que caracteriza al hombre barroco en Alemania.

El número de muertes en batalla y campañas prolongadas fue notablemente alto. Los civiles, que estaban en contacto directo con los ejércitos de ambos lados, veían cómo eran robados sus cosechas y ganados. Debido al mal abastecimiento de los ejércitos, los soldados practicaban el saqueo y la destrucción de las aldeas, pueblos y casas, prendían fuego a los cultivos, robaban los animales y depredaban las propiedades. Los comandantes no imponían disciplina. Lo peor era cuando tenían que repetir las campañas, porque los líderes militares no habían alcanzado sus objetivos anteriormente. Por lo tanto, algunas regiones estaban sujetas a una devastación continua, así como al hambre y al enfermedad traídas por los ejércitos.

A finales de 1633, los primeros soldados españoles llegaron a Andechs en la Alta Baviera para ayudar al emperador Fernando II en la lucha contra los suecos y los príncipes luteranos. Los regentes veían la llegada de los soldados como un medio para reorganizar las circunstancias de la guerra; pero la población local experimentaba el estallido de desorden con la llegada de las tropas. Ambas perspectivas son discordantes y, sin embargo, están relacionadas.

Esta observación da el primer indicio de las contradicciones del violento orden impuesto a principios del siglo XVII, y de la formación de los estados nacionales: orden puede significar desorden, si cambiamos la perspectiva del observador ${ }^{7}$.

\footnotetext{
7 Ansgar Cordie, Raum und Zeit des Vaganten. Formen der Weltaneignung im deutschen Schelm (Berlín: Walter de Gruyter, 2001), 37: «Diese Beobachtung gibt einen ersten Hinweis auf die Widersprüche der gewaltsam durchgesetzten Ordnung, die zu Beginn des 17. Jahrhunderts das Entstehen der
} 
Uno de los ciudadanos de Andechs, Mauro Friesenegger, escribió un diario en el que describió las contradicciones del espectáculo: mientras la gente caminaba «con sus cuerpos semidesnudos y magros, como figuras que usan la máscara del hambre y del sufrimiento; los oficiales estaban vestidos de ricos y pomposos» ${ }^{8}$. El comportamiento de los soldados era resultado de la corrupción rampante permitida por la situación de guerra. Incluso actuando en defensa del Imperio de los Habsburgo -católicos-, los soldados saqueaban y devastaban las iglesias. En Baviera, en circunstancias normales, delitos de este tipo significarían un ataque contra el orden sagrado y serían castigados severamente. De acuerdo con el diario de Friesenegger «tan pronto ellos [los soldados] entraron en la iglesia, se llevaron todo lo que estaba allí. Utilizaron la casa de Dios como una taberna (...)»? . Esto revela la corrupción del papel del ejército, cuya función principal debería ser el uso de la autoridad para ordenar y regular, y no para perturbar el orden, o crear una atmósfera caótica y grotesca.

Las manifestaciones de lo grotesco han recibido diferentes interpretaciones a lo lardo del tiempo. Uno de los autores más importantes en el tratamiento de este tema fue Wilhelm Kayser, que en 1957 publicó Das Groteske. Seine Gestaltung in Malerei und Dichtung $^{10}$. Kayser definió que uno de los rasgos esenciales de lo grotesco es la presentación de paradojas y contradicciones, responsables de revelar que nuestro mundo, confiable y aparentemente ordenado, se pierde en la irrupción de potencias abismales, se desmantela en sus formas y se disuelve en sus ordenaciones. Por lo tanto, las manifestaciones grotescas despiertan «una angustia desconcertada ante la aniquilación del mundo» porque la obra de arte tiene «una relación subterránea con nuestra realidad ${ }^{11}$. Un buen ejemplo, de acuerdo con Kayser, es la arte de Pieter Brueghel, el Viejo. Este pintor, que supo retratar figuras infernales como el Bosco, las insertó en el mundo familiar. Es precisamente la inclusión de lo abismal y fantasmal en un universo aparentemente ordenado y armonioso lo que produce el contraste y despierta lo grotesco ${ }^{12}$.

En la Guerra de los Treinta Años, lo grotesco surge precisamente de los contrastes entre lo que debería ser y lo que nace de la apariencia de autoridad y de orden que no se concretizan: los miembros del ejército «llegaron para ayudan», pero se comportan como si fuesen los enemigos de la gente. De la revelación de esta cruel

Territorialstaaten ermöglichte: Ordnung kann zugleich Unordnung bedeuten, wenn der Standpunkt des Beobachters wechselt».

${ }^{8}$ Marius Friesenegger, Tagebuch aus dem dreißigjährigen Krieg, nach einer Handschrift im Kloster Andechs (München: Süddeutscher Verlag, 1974), apud. Cordie, 2001, 37.

${ }^{9}$ Ibídem.

${ }^{10}$ Wolfgang Kayser, O Grotesco. Configuração na pintura e na literatura (São Paulo: Perspectiva, 1986).

${ }^{11}$ Kayser, 1986, 36. De acuerdo con Kayser, la forma más exacta para definir el concepto es a través de las artes. Sin embargo, también examinó una serie de obras literarias, tales como los cuentos fantásticos de E.T.A. Hoffmann, Gottfried Keller, comedia del arte, Sturm und Drang, etc.

${ }^{12}$ Lo mismo se pasa con la obra Las Meninas (1956) de Velázquéz: mientras un grupo de pequeñas señoras elegantes del palacio están llenas de solemnidad, en primer plano a la derecha podemos ver dos damas de la corte deformes. El contraste es aún más grave, porque parecen formar parte de una corte que debería ser completamente sublime. 
verdad -que es resultado de la lógica de la guerra- surge lo grotesco. A medida que la guerra continúa, más inhumanos y animalizados se vuelven los hombres. La pobreza, la falta de alimentos y provisiones, significaba que, cada vez más, las personas perdían la cortesía y se dedicaban a actividades bajas: la guerra suspendió las reglas de buena conducta.

Cuando Stengel publicó el texto de Bidermann, estaba inmerso en este escenario de la Guerra de los Treinta Años; cuando Hörl hizo su traducción, enfrentaba las consecuencias dejadas por el conflicto. En ambas situaciones reinaban la pobreza, la crueldad, la inseguridad, el caos. El orden humano -en el cual hay bondad, generosidad, respeto, cumplimiento de reglas y promesas- había dejado de existir. El mundo estaba vuelto del revés.

Al entrar en el país del Carnaval, los viajeros alemanes se enfrentan con «intimidantes y extraños enmascarados», vestidos como monstruos, mitad bestias y mitad hombres. Las máscaras de Carnaval eran parte de la esencia de esta fiesta. En el Carnaval, especialmente en el sur de Europa, en la Edad Media, al lado del mundo oficial había un «segundo mundo», al cual pertenecían los hombres durante un cierto período de tiempo. El Carnaval era una «segunda vida», porque representaba el mundo «al revés»: se bebía y se comía demasiado, se suspendían las reglas de buena conducta, se invertían los roles de las relaciones sociales; se profanaban las figuras de autoridad y jerarquía, etc. Era un período privilegiado, en el cual lo que se pensaba podría ser expresado con relativa impunidad. El uso de máscaras «ayudaba a la gente a liberarse de su ser cotidiano dando a cada uno la sensación de impunidad como el manto de la invisibilidad ${ }^{13}$. La fiesta funcionaba como una "válvula de seguridad" y esta experiencia de éxtasis era seguida por el retorno sobrio a la realidad cotidiana, a la estructura social normal.

En este sentido, la fiesta popular del Carnaval era muy positiva. El Carnaval significaba la renovación. La transformación grotesca del cuerpo y las manifestaciones grotescas - la relegación de lo más alto, el uso de máscaras y disfraces, la embriaguez, la gula y el sexo, el intercambio de roles y estatus social, la violencia física y verbal, el lenguaje profano, la risa y la burla-, promovían el renacimiento: de la destrucción del mundo conocido renace el mundo civilizado ${ }^{14}$.

Es fácil ver que estos rasgos positivos del Carnaval y de lo grotesco no están presentes en Utopía o en Bacusia. Lo grotesco, las risas, las máscaras y la locura se mantienen, pero ya no son lúdicos y alegres. La renovación no sucede en este lugar imaginario, porque la fiesta es ininterrumpida. No hay vuelta a la cruda realidad, a la estructura social normal. Después del Carnaval, no hay Cuaresma, porque el Carnaval es eterno. Lo grotesco presentado en el país del Carnaval se transforma en «vida

${ }^{13}$ Peter Burke, Cultura Popular na Idade Média (São Paulo: Companhia das Letras, 2010), 225.

14 «Die bestehende Welt wird zerstört, um sich in einer neuen Geburt zu erneuern. Die Welt gebiert sterbend». Mikhail Bakunin, Literatur und Karneval. Zur Romantheorie und Lacbkultur (Múnich: Fischer, 1969), 26. 
inferior»y parece estar destinado a asustar al lector. Las mascaras ocultan, mienten, inducen a error y provocan distanciamiento. El horror surge de la falta de perspectiva de renovación, de la falsa promesa de celebración y alegría. La eterna combinación de «comer, beber y jugar»-ocupaciones principales de la gente del país del Carnaval-y el odio a los libros, al conocimiento y a los estudios -los libros son prohibidos en este lugar- es aterradora precisamente a causa de su condición de eternidad. La risa del pueblo es monstruosa y cruel, y las mascaras son lúgubres debido a la falta de perspectivas de un futuro, por su condenación a la locura eterna. Por lo tanto, los viajeros alemanes y el público lector se darán cuenta de que en la ciudad de la euforia eterna no hay alegría, sino sólo la horrible comedia de la teratología. La ciudad que rechaza el amor a los estudios y al conocimiento no es feliz.

\section{EL GÉNERO LITERARIO UTÓPICO}

Raymond Trousson, en el libro Voyages aux pays de nulle part. Histoire de la pensée utopique $(1975)^{15}$, pone en primer lugar la dificultad de definir las características del género utópico. La palabra "utopía" floreció en todos los campos -ciencia, literatura, política y sociología-, abarcando un conglomerado de definiciones y conceptos heterogéneos. En general, en lenguaje común y popular, es sinónimo de ilusorio e inalcanzable; y el utopista parece ser alguien que ignora la realidad y la dinámica social. Sin embargo, la utopía nace como algo muy diferente de eso. En 1516, Tomás Moro

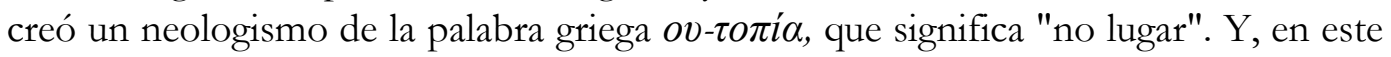
no-lugar, el autor observó los males de realidad de su tiempo y trató de crear soluciones para ella.

En la introducción a la obra de Trousson en italiano, publicada en 1992, Vita Fortunati concluye:

Il mondo dell'utopia è quello della possibilità: ogni costruzione utópica parte da un 'se' iniziale (...). Il "mode utopique" si caratterizza quindi come la facoltà di immaginare, di modificarei 1 reale com l'ipotesi di creare un ordine diferente dal reale ${ }^{16}$.

Por esta razón, sólo se puede entender una obra de género utópico cuando nos fijamos en su entorno histórico, político, religioso y cultural. La Utopía de Moro cristaliza en otro mundo un momento de reflexión sobre la realidad.

${ }^{15}$ Se utiliza la traducción italiana de Raffaela Medici (1992).

16 Vita Fortunati, "Introduzione“, en Raymond Trousson, Viaggi in nessun luogo. Storia letteraria del pensiero utópico (Ravenna: Longo, 1992), 5-12, 6. Trousson, 1992, p. 6. Trousson se basa en las ideas de Raymond Ruyer, en L'utopie et les utopies (1950), para decir que el método utópico es una característica fundamental del pensamiento humano, que consiste en la capacidad de imaginar posibilidades para la realidad. La utopía es un ejercicio mental, un método de especulación heurística. Trousson, 1992, pp. 17-18. 
André Prévost, en "La Utopía: el género literario" (1971) ${ }^{17}$, establece que la obra utópica parece, a primera vista, ser algo modesto, como recuerdos de viaje. Sin embargo, el viaje de Hitlodeo a Utopía es un poderoso instrumento de crítica social, ya que presenta dos paisajes: en el segundo libro, la isla de Utopía; en el primero, la vieja Europa, que Hitlodeo contrasta con la "isla feliz". En la primera, "una realidad distópica", las locuras y desequilibrios del absurdo sistema europeo, la sangrienta batalla de Blackheat (1497), la invasión de Italia por el rey de Francia, la anexión de las posesiones borgoñonas y flamencas, los campesinos pobres expulsados de la tierra para criar ovejas, la condena de 73.000 personas a la horca por Enrique VIII, etc. Todo ello suena «como una antítesis (...) de la utopía» según Prévost:

Si leemos las páginas de la vida de los utopianos después de las relacionadas con el mismo tema en Distopía, podemos ver con qué precisión los medicamentos se corresponden con el diagnóstico (Prévost, 2015, 440) ${ }^{18}$.

$\mathrm{Al}$ viajar a un lugar ficticio donde se purifican las costumbres y los males son resueltos, Hitlodeo puede transmitir a los lectores una nueva sabiduría, un posible orden mundial de armonía y felicidad colectiva. «La utopía es un arte consciente de sí mismo» (Ibidem). El género utópico, tal como lo practica Moro, requiere que el lector mantenga su inteligencia perfectamente lúcida: «la utopía es un ejercicio de la inteligencia tanto como un juego de la imaginación», ya que hace uso de la «ironía utópica», diciendo lo contrario de lo que piensa, «el trabajo utópico quiere comunicar una verdad profunda» (Ibídem). Por esta razón, a menudo para indicar que no está haciendo simplemente una fábula, el autor hace guiños para el lector cómplice, utilizando palabras enigmáticas, rompecabezas y conceptos autodestructivos ${ }^{19}$. La palabra «utopía» es uno de ellos, ya que evoca el absurdo, «el país que no existe». Amaurota, la capital de la isla de Moro es la «ciudad invisible»; Anhídrido es el río sin agua.

Una primera lectura de la utopía de Bidermann muestra que el autor no creó un mundo en donde se solucionaran los males de su tiempo; y que el país del Carnaval no es un espejo ideal de su propio mundo. Por el contrario: el jesuita retrató un universo fantasmal, desordenado y grotesco, puso el foco sobre los vicios, sus excesos y las nefastas consecuencias de un mundo no guiado por la Iglesia Católica. Sin embargo, de manera similar al Libro I de la Utopía de Moro, el jesuita llamó la atención sobre lo absurdo de su realidad: en el siglo XVII, Europa parecía estar vuelta del revés, era «una nave de locos»: la religión justificaba masacres, todos vivían bajo el signo de la muerte, los países católicos apoyaban a los calvinistas contra el emperador católico;

17 André Prévost, “A Utopia: o gênero Literário”, Morus - Utopia e Renascimento 10 (2015): 437-447.

${ }_{18}$ Para Prévost, «se forjó (...) la palabra Distopía para llamar a este desorden mundial, el país de sufrimiento y miseria: las distorsiones sociales, económicas y políticas se muestran por todas partes; (...) la ambición de los reyes (...) la pobreza, la vagancia, el robo; la ignorancia y la necedad de los sacerdotes les hacen incapaces de renovar las costumbres; los sistemas económicos, financieros y políticos, la propiedad privada y su base, el oro, las fuentes de todos los males, todos los estragos». Prévost, 2015, 440.

${ }^{19}$ Según Prévost, Moro utilizó cerca de 20 palabras enigmáticas. 
soldados imperiales invadían y saqueaban pueblos, etc. Llamar Utopía a una obra que trata sobre el viaje al país grotesco del Carnaval es irónico. El país del Carnaval no es una «isla feliz», no hay orden, armonía o felicidad colectiva, sino todo lo contrario. Llamarlo «Utopía» es lo mismo que llamar a un río «anhídrido». Al igual que el río, que evoca el absurdo de un río sin agua, la felicidad eterna prometida en la Utopía de Bidermann está hecha de una alegría furiosa, dominada por el miedo, por lo grotesco, por la locura.

La locura del mundo, típica de las expresiones literarias y artísticas del Barroco, es reflejo del desorden y de la inestabilidad que sacudió a Europa en el siglo XVII. Según Maravall, «cuando el hombre barroco habla del "mundo loco", traduce toda una serie de experiencias concretas» del trastorno y de la confusión general ${ }^{20}$. El orden tradicional del universo ya no existe. «Por lo tanto, otro tema importante revitalizado por el Barroco es el mundo al revés». ${ }^{21}$ Para Maravall, el mundo al revés que se plantea en este momento histórico es «producto cultural de una sociedad en proceso de cambio» $^{22}$ y de la sensación de inestabilidad que se produce a la vista de un trastorno tambaleante. Y el mundo al revés retratado en el país del Carnaval, variación de género utópico en el sentido de que es producto de la imaginación de otro mundo ${ }^{23}$, no aspira a un estado de orden y felicidad como Moro; por el contrario: el mundo al revés no respeta las reglas del realismo narrativo, confiesa su improbabilidad y, sobre todo, no ofrece un universo alternativo. La utopía es constructiva e imagina posibilidades, mientras que el mundo al revés destruye la jerarquía social y todas las formas de $\operatorname{orden}^{24}$.

Bidermann llama a su país del Carnaval Utopía y confesa su improbabilidad. Su Utopía no es un mundo que soluciona los problemas contemporáneos al autor, ni un espejo ideal de su vida cotidiana. Pero en la extraña imagen que refleja el espejo distorsionado del país del Carnaval, se le puede atribuir un carácter utópico, en el sentido de que Bidermann, Stengel y Hörl - del mismo modo que Moro y otros utopistas- discuten hábilmente problemas de su tiempo en un lugar imaginario, en ningún lugar, en una utopía. El país del Carnaval, como obra utópica al revés, permite que nos aproximemos al ambiente Barroco y contrarreformista. El país del Carnaval es un mundo fantasmagórico y grotesco en donde cualquier orden parece desarticularse. Un rasgo esencial de lo grotesco es que no consiste en su propio dominio y en un fantasear totalmente libre (que no existe). El mundo grotesco es nuestro mundo y no lo es. Lo grotesco tiene su fundamento precisamente en la experiencia de que nuestro mundo, confiable y aparentemente ordenado, se pierde por la irrupción de potencias abismales, se desmantela en sus formas y se disuelve en sus estatutos. Las representaciones de la ininterrumpida celebración carnavalesca del país del Carnaval adquieren relación con

\footnotetext{
${ }^{20}$ José Antonio Maravall, A Cultura do Barroco (São Paulo: Edusp, 1997), 248-249.

21 Ibídem, 249.

22 Ibídem, 251.

23 Para Trousson, la utopía se centra en la construcción de un lugar distinto. Trousson, 1992, 7.

${ }^{24}$ Ibídem, 25. El procedimiento del mundo al revés es adoptado por Aristófanes en La Asamblea de las mujeres (391 a.C.); por Rabelais en la abadía de Telema (1534); en la Cucaña; etc.
} 
la realidad y, por lo tanto, permiten el acercamiento del lector con la cultura barroca de la Alemania del siglo XVII.

\section{BIBLIOGRAFÍA}

Bakunin, Mikhail, Literatur und Karneval. Zur Romantheorie und Lachkultur (Múnich: Fischer, 1969).

Burke, Peter, Cultura Popular na Idade Média (São Paulo: Companhia das Letras, 2010).

Burkhardt, Jacob, Der Dreißigjährige Krieg (Frankfurt: Surkamp, 1992).

Carpeaux, Otto Maria, História concisa da literatura alemã (São Paulo: Faro, 2013).

Cordie, Ansgar, Raum und Zeit des Vaganten. Formen der Weltaneignung im deutschen Schelm (Berlín: Walter de Gruyter, 2001).

Friesenegger, Marius, Tagebuch aus dem dreißigjährigen Krieg, nach einer Handscbrift im Kloster Andechs (Múnich: Süddeutscher Verlag, 1974).

Kayser, Wolfgang, O Grotesco. Configuração na pintura e na literatura (São Paulo: Perspectiva, 1986).

Le Goff, Jacques, Os Intelectuais na Idade Média (São Paulo: Editora Brasiliense, 1989).

Maravall, José Antonio, A Cultura do Barroco (São Paulo: Edusp, 1997).

Prévost, André, “A Utopia: o gênero Literário”, Morus - Utopia e Renascimento 10 (2015): 437-447.

Schiller, Friedrich, Historische Scbriften und Eräählungen (Stuttgart: Deutscher Klassiker Verlag, 1879).

Schuster, Margrit, Jakob Bidermanns "Utopia": Ed. Mit Übersetzung und Monographie; nebs vergleichenden Studien zum beigedr. Plagiat d. Christoph Andreas Hörl von Wattersdorf (,Bacchusia oder Fassnacht-Land“) (Berna: Peter Lang, 1984).

Trousson, Raymond, Viaggi in nessun luogo. Storia letteraria del pensiero utópico (Rávena: Longo, 1992). 
Recibido: 3 de abril de 2017 Aprobado: 25 de octubre de 2017 